\title{
Effect of Smad3/4 on chemotherapeutic drug sensitivity in colorectal cancer cells
}

\author{
SUNG UNG MOON ${ }^{1,2}$, MI HYUN KANG ${ }^{1,2}$, JI HEA SUNG ${ }^{1,2}$, JIN WON KIM ${ }^{1}$, JEONG OK LEE ${ }^{1}$, \\ YU JUNG KIM ${ }^{1}$, KEUN WOOK LEE ${ }^{1}$, SOO MEE BANG ${ }^{1}$, JONG SEOK LEE ${ }^{1}$ and JEE HYUN KIM ${ }^{1}$ \\ ${ }^{1}$ Division of Hematology and Medical Oncology, Department of Internal Medicine, \\ Seoul National University Bundang Hospital, Seoul National University College of Medicine; \\ ${ }^{2}$ Biomedical Research Institute, Seoul National University Bundang Hospital, \\ Bundang-gu, Seongnam-si, Gyeonggi-do 463-070, Republic of Korea
}

Received July 11, 2014; Accepted October 14, 2014

DOI: 10.3892/or.2014.3582

\begin{abstract}
Smad3 and Smad4 are signaling mediators in the transforming growth factor $\beta$ (TGF $\beta$ ) pathway and play a major role in the progression and migration of many types of cancers. The TGF $\beta$ pathway is correlated with resistance against both targeted and conventional chemotherapeutic drugs. The aim of this study was to determine the effect of Smad3/4 on drug sensitivity in chemotherapy-resistant colorectal cancer (CRC) cells. We isolated the TGF $\beta$-mediated chemoresistant CRC cell line DLD1-5FU-C10, which showed high expression of Smad3/4 and p21. In order to analyze the influence of Smad3/4 on drug sensitivity in DLD1-5FU-C10 cells, we knocked down Smad3/4 using small interfering RNAs (siRNA). The results showed similar drug sensitivity between the DLD1-5FU-C10 and the DLD1 control cells and reduced p21 expression. In addition, we found a significant increase in the levels of 3 TGF $\beta$ downstream factors: interleukin 6 (IL6), plasminogen activator (PLAU) and prostaglandin-endoperoxide synthase 2 (PTGS2). Furthermore, we showed that Smad3/4 regulated the JAK1/STAT3 pathway via IL6 in the chemoresistant CRC cell line. In conclusion, we identified Smad3/4 as a novel drug sensitivity regulator in TGF $\beta$-mediated chemotherapyresistant CRC cells. Our results suggest that Smad3/4 regulate p-STAT3 signaling by IL6 and p21 and highlight an important role for STAT3 signaling in Smad3/4 regulated drug sensitivity in chemoresistant CRC cells.
\end{abstract}

\section{Introduction}

Colorectal cancer (CRC) is a complex disease with characteristics such as sustained proliferation, cell death evasion

Correspondence to: Professor Jee Hyun Kim, Department of Internal Medicine, Seoul National University Bundang Hospital, 82 Gumi-ro 173 Beon-gil, Bundang-gu, Seongnam-si, Gyeonggi-do 463-070, Republic of Korea

E-mail: jhkimmd@snu.ac.kr

Key words: TGF $\beta$, Smad3/4, p21, IL6, JAK1/STAT3, colorectal cancer and tissue invasion and metastasis, which make treatment difficult $(1,2)$. Cancer cell migration and invasion are critical steps in the metastatic process and are regulated by numerous cancer-secreted factors which modify the cancer microenvironment by acting on stromal recruitment and extracellular matrix (ECM) degradation (3).

Transforming growth factor $\beta$ s (TGF $\beta \mathrm{s}$ ) are $25-\mathrm{kDa}$ growth factors that play a unique and central role in homeostasis, wound healing, fibrosis, angiogenesis, carcinogenesis and cell differentiation $(4,5)$. Each member of the TGF $\beta$ family is encoded by different genes, although they act through the same receptor-signaling cascade. They are stored in the ECM and attach to latent TGF $\beta$-binding proteins $(6,7)$. This attachment prevents the binding of the molecule to its receptor (8).

During breast tumor progression, the loss of TGF $\beta$ growth-inhibitory effects is frequently due to defects in c-myc and p15 regulation by TGF $\beta$ (9). However, other TGF $\beta$ responses are generally unrelated to growth inhibition and favor tumor progression and metastasis (10-14). Moreover, a study by Dai et al showed that p21 interacts with Smad3/4 and the acetyl transferase $\mathrm{p} / \mathrm{CAF}$ in order to regulate Smad transcriptional activity, as well as gene transcription of several other metastatic genes in breast cancer patients. These results highlight the importance of p21/p/CAF-induced breast cancer cell migration and invasion at the transcriptional level (15). In most CRC patients, TGF $\beta$ is overexpressed and is likely associated with poor survival (16). A recent study showed that high p21 expression in pretreatment biopsies was associated with poor prognosis in $\mathrm{CRC}$ patients treated with 5-fluorouracil (5-FU)-based chemoradiotherapy (17).

Signaling from TGF $\beta$ through a transmembrane serine-threonine kinase is an important $S \operatorname{mad} 3 / 4$ pathway, but plays an ambiguous role in carcinogenesis (18-22). The regulatory power of $\mathrm{Smad} 3$ as a transcriptional regulator is augmented or modulated by interactions with $~ 50$ co-transcription factors (23). In addition, a study by Ulloa et al reported a mechanism of transmodulation between the STAT and SMAD signal-transduction pathways (24).

Previous studies have highlighted the important role of TGF $\beta$ and Smads in various cancer types. However, the roles of Smads downstream of TGF $\beta$ are still unknown in CRC 
and their association with chemosensitivity has not been elucidated. The goal of this research was to investigate how Smad3/4 are correlated with chemotherapeutic drug sensitivity in human CRC and whether Smad3/4 and p21 are required to promote human CRC cell progression by TGF $\beta$ signaling.

\section{Materials and methods}

Cell culture and reagents. The DLD-1, SNU-175, SNU-C4, Colo-320M, HT-29 and HCT-15 human CRC cell lines were obtained from the Korean Cell Line Bank (KCLB). DLD-1, SNU-175, SNU-C4, Colo-320M, HT-29 and HCT-15 were cultured in RPMI-1640 medium containing $10 \%$ fetal bovine serum (FBS). All cells were cultured in a humidified incubator at $37^{\circ} \mathrm{C}$ with $5 \% \mathrm{CO}_{2}$. DLD-1 was made resistant to $5-\mathrm{FU}$ by incremental and continuous exposure to a formulation of 5-FU and TGF $\beta 1$. Initially DLD-1 cells were treated with $10 \mu \mathrm{M}$ $5-\mathrm{FU}$ and $5 \mathrm{ng} / \mathrm{ml}$ TGF $\beta 1$ by limiting dilution. The resulting chemoresistant CRC clone, named DLD1-5FU-C10, was able to grow in the presence of $75 \mu \mathrm{M}$ of 5-FU in culture medium.

Cell viability inhibition by cytotoxic agents. The CRC cell lines were seeded at $3 \times 10^{3}$ cells/well in 96-well white flat-bottomed plates. After incubation for $24 \mathrm{~h}, \mathrm{CRC}$ cells were treated with 5-FU or oxaliplatin at various concentrations $(0$, $10,100$ and 1,000 or $0,0.3,0.6,6,12,120$ and $250 \mu \mathrm{M})$ in $10 \%$ FBS-supplemented RPMI-1640 for $72 \mathrm{~h}$. The toxicity of these treated cells was measured by adding $100 \mu \mathrm{l}$ of CellTiter-Glo ${ }^{\circledR}$ reagent (Promega, Madison, WI, USA) to each well. Luminescence values in each well were determined using a Spectra MAX plate reader (Molecular Devices, Sunnyvale, CA, USA). Luminescence values from wells without cells (background) were subtracted from the values of the wells with cells. Data were analyzed with SigmaPlot software (Systat Software Inc., Chicago, IL, USA) using Logistic 3 parameter analysis to determine the half-maximal inhibitory concentration $\left(\mathrm{IC}_{50}\right)$ of the chemotherapeutic agents.

Reverse transcription-polymerase chain reaction (RT-PCR). Total RNA was extracted from the cells using TRIzol reagent (Invitrogen, Grand Island, NY, USA) and reverse transcribed into cDNA using the High Capacity RNA-to-cDNA kit (Applied Biosystems, Grand Island, NY, USA) according to the manufacturer's instructions. The primer sequences were: interleukin 8 (IL8) forward, GCAGAGGCCACCTGGATTG TGC and reverse, TGGCATGTTGCAGGCTCCTCAGAA; (IL6) forward, CTCCCCTCCAGGAGCCCAGC and reverse, GCAGGGAAGGCAGCAGGCAA; plasminogen activator (PLAU) forward, GCCCTGGTTTGCGGCCATCT and reverse, CGCACACCTGCCCTCCTTGG; matrix metalloproteinase 9 (MMP-9) forward, TGGACACGCACGACGTCT TCC and reverse, TAGGTCACGTAGCCCACTTGGTCC; prostaglandin-endoperoxide synthase 2 (PTGS2) forward, AGCTTTCACCAACGGGCTGGG and reverse, AAGACCT CCTGCCCCACAGCAA; p21 forward, TGTCCGCGAGGA TGCGTGTTC and reverse, GCAGCCCGCCATTAGCGCAT; GAPDH forward, GCCTCAAGATCATCAGCAATGCCT and reverse, TGTGGTCATGAGTCCTTCCACGAT; Smad3 forward, GGTCAAGAGCCTGGTCAAGA and reverse, TTG AAGGCGAACTCACACAG; Smad4 forward, GACTGAGG
TCTTTTCCGTTGG and reverse, CTTCAAGCTCTGAGCC ATGC; STAT3 forward, GTGGGCGAGCGGTGTTCTG and reverse, CAGAACACCGCTCGCCCAC; JAK1 forward, CAT GGTGGAAGAGTTTGTGGAA and reverse, CAGCTGTTT GGCAACTTTGAATT. The amplification conditions consisted of an initial denaturation at $95^{\circ} \mathrm{C}$ for $5 \mathrm{~min}$, then 40 cycles of denaturation at $95^{\circ} \mathrm{C}$ for $30 \mathrm{sec}$, annealing at $58^{\circ} \mathrm{C}$ for $30 \mathrm{sec}$ and elongation at $72^{\circ} \mathrm{C}$ for $30 \mathrm{sec}$. A $1 \%$ agarose gel, containing Loading Star (DyneBionc, Gyeonggi, Korea) for visualization, was run in Tris Borate-EDTA (TBE) buffer for $20 \mathrm{~min}$ at $100 \mathrm{~V}$, and the PCR products were analyzed using a Bio Image Analyzer (Fisher Scientific, Seoul, Korea).

Small interfering RNA (siRNA). DLD1-5FU-C10 cells were transfected with different Smad3 and Smad4 siRNAs (AccuTarget $^{\mathrm{TM}}$ Custom Designed siRNA; Bioneer, Daejeon, Korea) and comprised the following targeting sequences: Smad3 siRNA sense, 5'-GGAGAAAUGGUGCGAGAA Gtt-3'; Smad3 siRNA antisense, 5'-CUUCUCGCACCAUUU CUCCtc-3'; Smad4 siRNA sense, 5'-GGUGGAGAGAGUGA AACAUtt-3'; and Smad4 siRNA antisense, 5'-AUGUUUCAC UCUCUCCACCtt-3'. For transient transfections, $10^{5}$ cells were transfected with $100 \mathrm{nM}$ siRNA using Lipofectamine (Invitrogen).

Immunoblotting analysis. CRC cell lines and siRNA-transfected cells were collected and lysed with Cell Lysis Buffer (Cell Signaling Technology, Boston, MA, USA). Protein concentrations were determined using a Pierce BCA protein assay kit (Thermal Scientific Inc., Odessa, TX, USA). Equivalent amounts of protein from each lysate were separated using SDS-PAGE and were transferred to nitrocellulose membranes for immunoblotting. The membranes were washed 3 times with Tris-buffered saline (TBS) containing $0.1 \%$ Tween-20 (TBST). After blocking with TBST containing 5\% nonfat milk for $1 \mathrm{~h}$, the membranes were incubated with the appropriate primary antibody in TBST containing 3\% skin milk at $4^{\circ} \mathrm{C}$ overnight. All of the primary antibodies were diluted in an appropriate concentration of 3\% skim milkcontaining TBST. After treatment with the primary antibodies against Smad3, Smad4, p21 and IL-6 (all from Cell Signaling Technology), IL-8 and PLAU (both from Abcam, Cambridge, MA, USA), MMP-9, PTGS2, JAK1, STAT3 and $\beta$-actin (all from Cell Signaling Technology), the membranes were washed 3 times with TBST for $30 \mathrm{~min}$, followed by goat anti-rabbit or anti-mouse IgG-horseradish peroxidase-conjugated secondary antibody (diluted at 1:4,000) for $2 \mathrm{~h}$ at room temperature and washed 3 times with TBST for $1 \mathrm{~h}$. The membranes were developed using the ECL western blotting substrate (Promega) according to the manufacturer's instructions.

Cell migration assay. Cells were transfected with 3 siRNAs (negative siRNA, Smad3 siRNA and Smad4 siRNA) and plated in 6-well plates at $10^{6}$ cells/well. The 6-well plates ensured that images of the wound could be automatically captured at the exact same location by the Tsview 7 (Tucsen, Fuzhou, China). Cells were scratched using a cell scraper (SPL, Pocheon, Korea) to generate $\sim 250 \mu \mathrm{m}$-width wounds. After wounding, cells were washed 2 times with PBS and 5-FU was added in the presence or the absence of $5 \mathrm{ng} / \mathrm{ml}$ of TGF $\beta$. The plates 


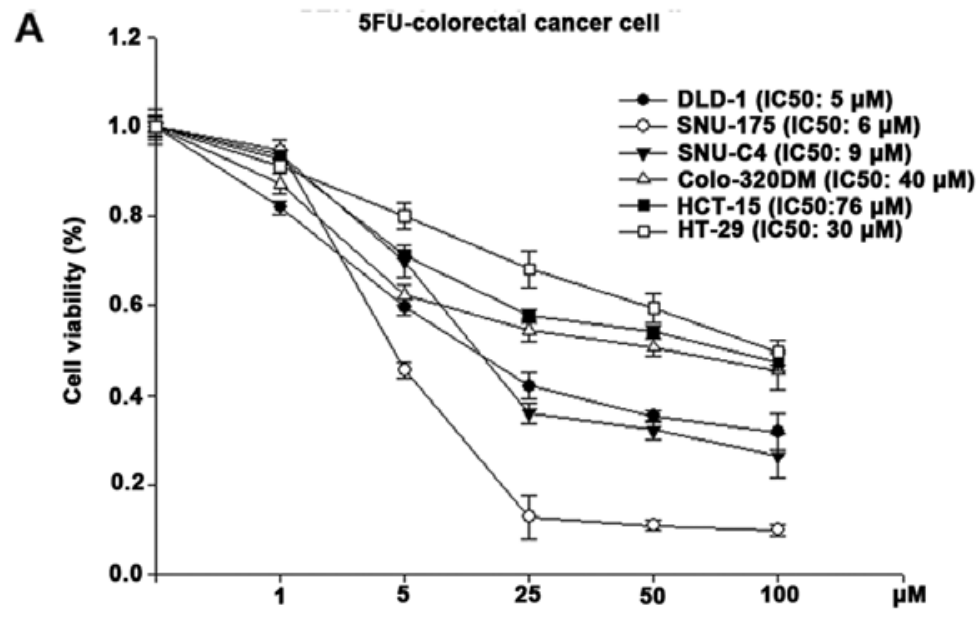

B

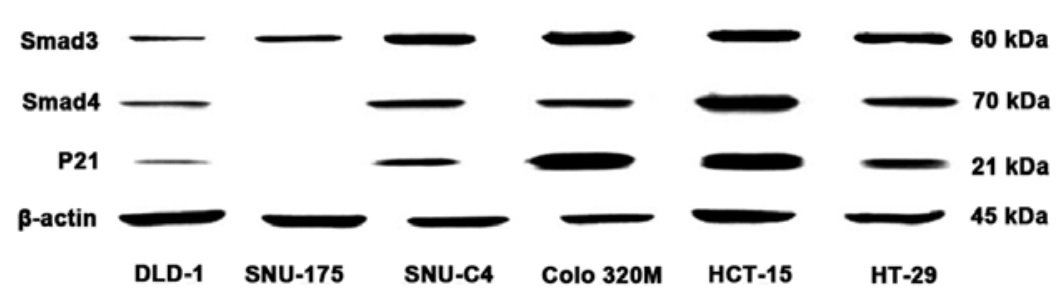

Figure 1. Cytotoxic effect of 5-fluorouracil on human colorectal cancer (CRC) cell lines as related to Smad3/4. (A) The cell viability of 3 CRC cell lines (DLD-1, SNU-175 and SNU-C4) showed high 5-fluorouracil (5-FU) sensitivity, whereas the other cancer cell lines (Colo-320M, HCT-15 and HT-29) showed relatively low 5-FU sensitivity. (B) High 5-FU-sensitive human CRC cell lines (DLD-1, SNU-175, SNU-C4) showed low Smad3/4 and p21 protein expression. The same lysates were also used to evaluate the expression of $\beta$-actin as the loading control.

were then placed into a Tsview 7 for $72 \mathrm{~h}$. The data were analyzed by wound width or relative wound width automatically measured by TSView 7 software (Tucsen).

Immunolocalization studies. CRC cell lines $\left(10^{5} / \mathrm{ml}\right)$ in $24-w e l l$ plates (Corning Inc., Corning, NY, USA) were washed 3 times with PBS, fixed with $100 \%$ ethanol for $10 \mathrm{~min}$ on ice and then washed 3 times with PBS. Cells were permeabilized with $0.025 \%$ Triton and blocked for $1 \mathrm{~h}$ at room temperature with dilution buffer (Invitrogen). Primary antibodies anti-Smad3, anti-Smad4 and anti-p21 (all from Cell Signaling Technology) were then added to the dilution buffer and incubated for $24 \mathrm{~h}$ at $4{ }^{\circ} \mathrm{C}$. The primary antibodies were removed and the cells were washed 3 times for 3 min each with PBS. Next, the cells were incubated with the appropriate secondary antibody prepared in dilution buffer conjugated to FITC (1:500) for $4 \mathrm{~h}$ at room temperature. Cells were washed again 3 times for 3 min each with PBS and the cells were visualized using a Zeiss Observer Z1 AX10 (ZEISS, Oberkochen, Germany) fluorescence microscope.

\section{Results}

Anticancer drug sensitivity of human CRC cell lines related to Smad3/4. The cytotoxic effects of 5-FU on 6 human CRC cell lines (DLD-1, SNU-175, SNU-C4, Colo-320M, HCT-15, HT-29) were examined using a luminescence assay (Fig. 1A). The $\mathrm{IC}_{50}$ values for 5-FU were 5, 6, 9, 40, 76 and $30 \mu \mathrm{M}$ in the DLD-1, SNU-175, SNU-C4, Colo-320M, HCT-15 and HT-29 cells, respectively. The cell viability of the DLD-1, SNU-175 and SNU-C4 cell lines reflected high sensitivity to 5-FU. The other cancer cell lines (Colo-320M, HCT-15, HT-29) showed relatively low 5-FU sensitivity. The protein expression levels of Smad3, Smad4 and p21 in the 6 human CRC cell lines were determined by immunoblotting (Fig. 1B). Although all of the cancer cell lines showed detectable levels of Smad3, Smad4 and p21, higher levels of Smad3/4 and p21 protein were noted in the Colo-320M, HCT-15 and HT-29 cells, which were the cell lines that showed decreased 5-FU sensitivity (Fig. 1B). Since the DLD-1 cells showed low Smad3/4 and p21 expression and high 5-FU sensitivity, we selected DLD-1 cells to proceed with the experiments and established resistance to 5-FU by TGF $\beta$ treatment (DLD1-5FU-C10 cells).

Isolating chemoresistant human CRC cells by TGF $\beta$ treatment. To confirm the cell viability to 5-FU in the DLD1-5FU-C10 cells, we analyzed intrinsic sensitivity to 5-FU, which resulted in a calculated $\mathrm{IC}_{50}$ value of $112 \mu \mathrm{M}$ for 5-FU (Fig. 2A). In addition, DLD1-5FU-C10 cells showed decreased sensitivity to oxaliplatin, a platinum-based antineoplastic agent, with a calculated $\mathrm{IC}_{50}$ of $137 \mu \mathrm{M}$ (Fig. 2B). Finally, we isolated DLD1-5FU-C10 cells that showed $\mathrm{IC}_{50}$ values $>10$-fold higher than the DLD1 control.

Smad3/4 are related to drug sensitivity and cell mobility via $p 21$. To evaluate further the relationship between Smad3/4 and drug sensitivity, Smad3/4 expression was knocked down by Smad3/4 siRNAs in the DLD1-5FU-C10 cells. The knockdown was confirmed by immunoblotting and RT-PCR (Fig. 3A). 

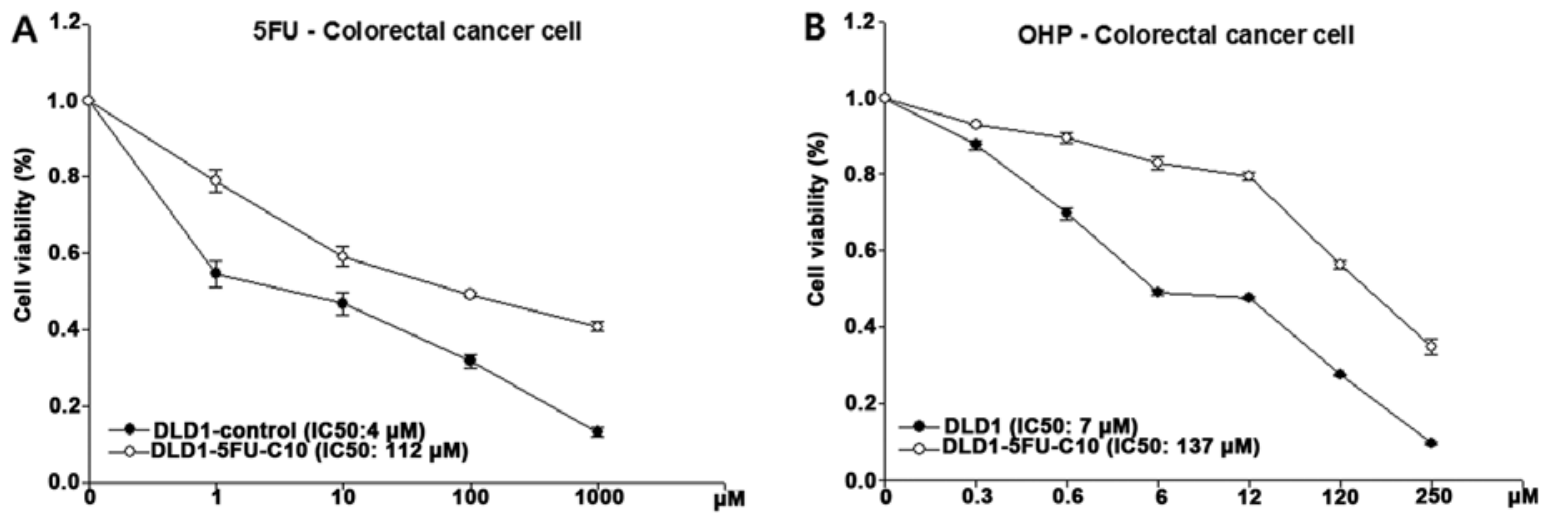

Figure 2. Cytotoxic effect of low drug sensitivity human colorectal cancer (CRC) cells mediated by TGF 3 . Low drug sensitivity human CRC cells were isolated by limiting dilution. The resulting clone, named DLD1-5FU-C10, was able to grow in the presence of $75 \mu \mathrm{M}$ of 5 -fluorouracil (5-FU) and $5 \mathrm{ng} / \mathrm{ml}$ of TGF $\beta$ in culture medium. DLD-1 and DLD1-5FU-C10 cells were treated with various concentrations of (A) 5-FU and (B) oxaliplatin (OHP) for $72 \mathrm{~h}$ and cell viability was determined using a cytotoxicity assay in each cell line.
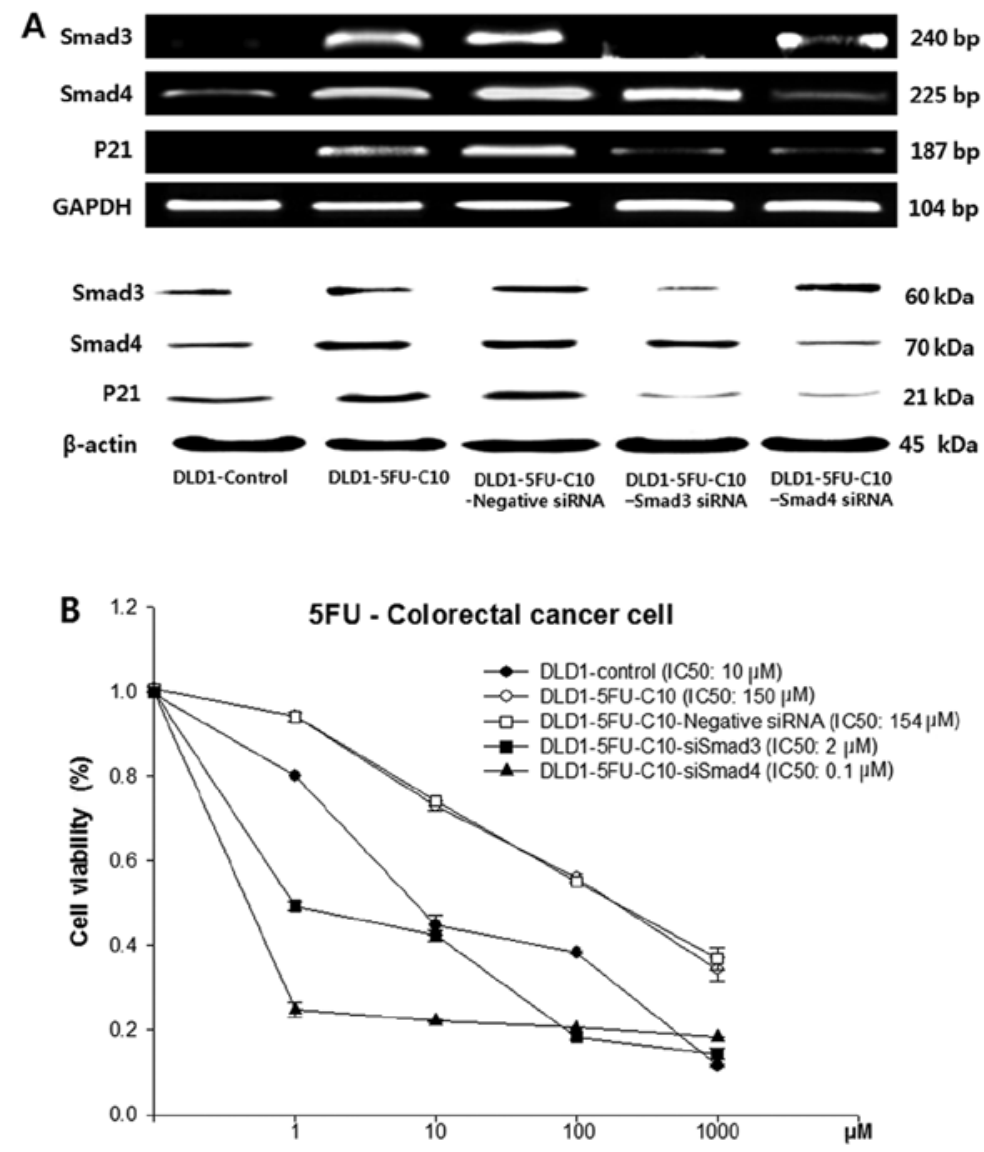

Figure 3. Smad3/4 are correlated with drug sensitivity in low drug sensitivity human colorectal cancer (CRC) cells. (A) Five groups of DLD-1 CRC cells (DLD-1 control, DLD1-5FU-C10, DLD1-5FU-C10-Negative siRNA, DLD1-5FU-C10-Smad3 siRNA, DLD1-5FU-C10-Smad4 siRNA) were analyzed. Reverse-transcription polymerase chain reaction (RT-PCR) and immunoblotting analysis for the expression of Smad3, Smad4, p21, GAPDH or $\beta$-actin were performed in the 5 groups of DLD1 CRC cells. The same lysates were also used to evaluate the expression of $\beta$-actin as a loading control. Data are representative of 3 independent experiments. (B) The DLD1-5FU-C10 cells with Smad3/4 knockdown showed lower viability than did the control DLD1-5FU-C10 cells. The 5 groups of DLD-1 CRC cells were treated with various concentrations of 5 -fluorouracil (5-FU) for $72 \mathrm{~h}$, and cell viability was determined using a cytotoxic assay in each cell line. When 5-FU was added in combination with Smad3/4 knockdown, cell viability was significantly lower than that of the DLD1-5FU-C10 cells.

$\mathrm{Smad} 3 / 4$ protein levels were decreased in the DLD1-5FU-C10 cells treated with Smad3 and Smad4 siRNA when compared with levels in the non-transfected DLD1-5FU-C10 cells or cells treated with the negative siRNA.
Smad3/4 siRNA caused slightly lowered p21 expression when compared with that in the non-transfected DLD1-5FU-C10 cells or the DLD1-5FU-C10 cells treated with the negative siRNA. Therefore, our results indicated that Smad3/4 down- 

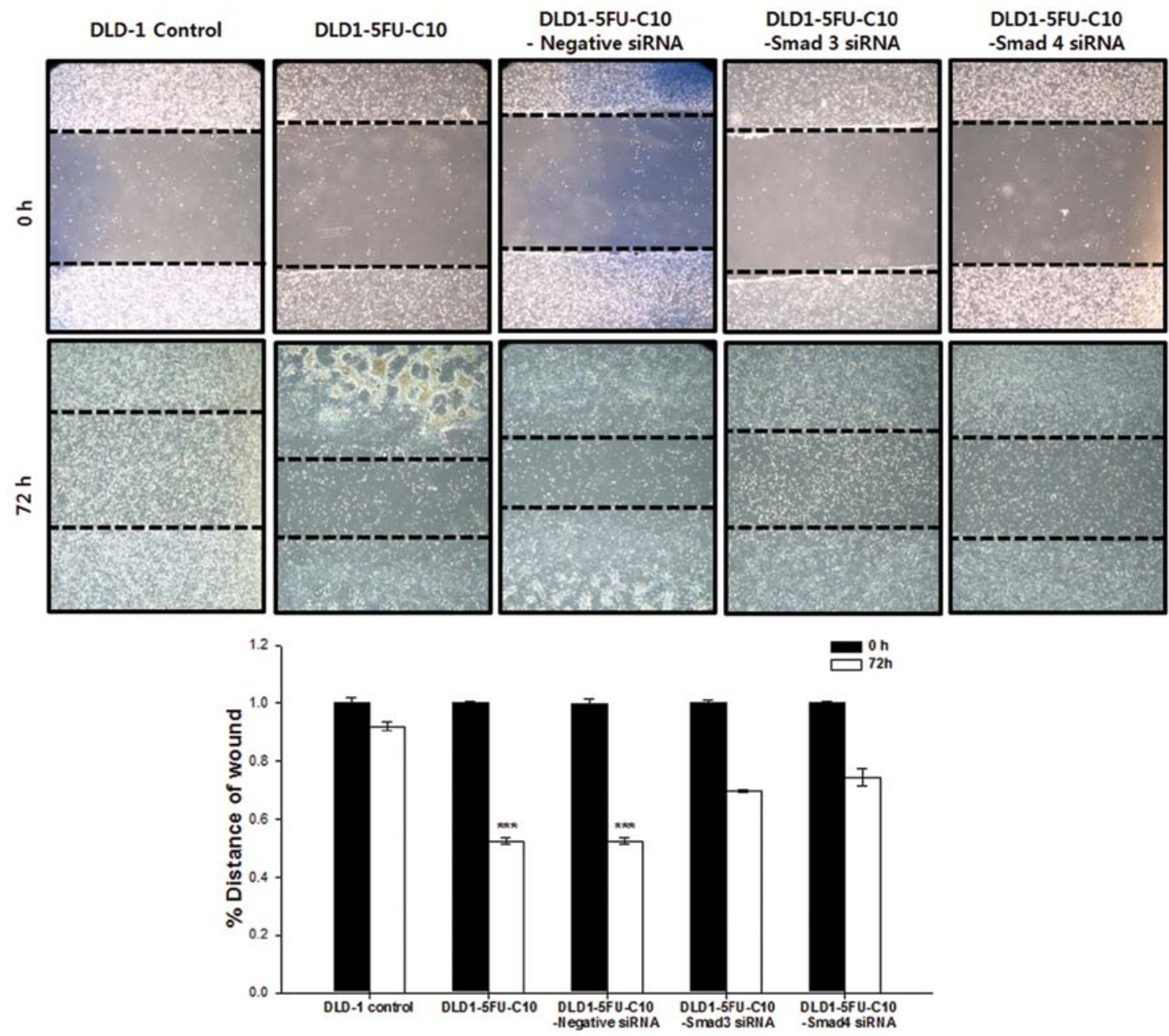

Figure 4. Smad3/4 are correlated with cell migration in low drug sensitivity colorectal cancer (CRC) cells mediated by TGF $\beta$. Five groups of DLD-1 CRC cells (DLD-1 control, DLD1-5FU-C10, DLD1-5FU-C10-Negative siRNA, DLD1-5FU-C10-Smad3 siRNA, DLD1-5FU-C10-Smad4 siRNA) were scratched and incubated for $72 \mathrm{~h}$. Wound closure was monitored by measuring wound widths. Magnification, $\mathrm{x} 40$. Data are means of triplicate samples, ${ }^{* * *}$ S.E. p $<0.0001$.

regulation reduced $\mathrm{p} 21$ expression in the DLD1-5FU-C10 cells. Knockdown of Smad3/4 expression in the DLD1-5FU-C10 cells led to a decrease in cell viability and $\mathrm{IC}_{50}$ values from $150 \mu \mathrm{M}$ for the DLD1-5FU-C10 cells to $0.1 \mu \mathrm{M}$ for the siSmad 4 cells and $2 \mu \mathrm{M}$ for the siSmad 3 cells (Fig. 3B).

We also investigated whether Smad3/4 are required for cell migration in the DLD1-5FU-C10 cells using the scratch/wound healing assay in the presence of 5-FU. Fig. 4 shows the migration of DLD1-5FU-C10 and DLD1-5FU-C10 cells with Smad3/4 knockdown (DLD1-5FU-C10-Smad3 siRNA, DLD1-5FU-C10-Smad4 siRNA). The rate of cell migration was significantly higher in the DLD1-5FU-C10 cells than that in the DLD1 control and Smad3/4 knockdown groups (Fig. 4). Wound closure was monitored by measuring wound widths.

Chemoresistant human CRC cells induce transcriptional activity of TGF $\beta$ downstream genes. Next, we performed signal pathway profiling experiments in chemoresistant human CRC cells (DLD1-5FU-C10), using transiently transfected Smad3/4 siRNA. We identified multiple Smad3/4-dependent TGF $\beta$ target genes, among which we selected those known to be associated with drug sensitivity. The shortlist included
5 candidate target genes from our literature search (15): IL6, IL8 (chemokine), PTGS2, PLAU and MMP-9. The 5 TGF $\beta$-induced downstream genes were detected by immunoblotting (Fig. 5A) and RT-PCR (Fig. 5B). As shown in Fig. 5, DLD1-5FU-C10 cells showed significantly higher mRNA expression of IL6, PLAU and PTGS2 than did the DLD1 control cells. Furthermore, we analyzed the influence of Smad3/4 knockdown on the DLD1-5FU-C10 cells, which showed a recovery-signaling pathway to the DLD1 control.

Smad3/4 regulate STAT3 signaling in chemoresistant human $C R C$ cells. We showed that $\mathrm{Smad} 3 / 4$ induced the protein kinase JAK1 and the transcription factor p-STAT3 in the chemoresistant human CRC cells (DLD1-5FU-C10) via TGF $\beta$ (Fig. 5). Furthermore, Smad3/4 knockdown in the DLD1-5FU-C10 cells decreased p-STAT3 signaling. Thus, we hypothesized that the JAK1/STAT3 pathway could act downstream of Smad3/4 to regulate anticancer drug sensitivity. We investigated the localization of $\mathrm{p} 21, \mathrm{Smad} 3 / 4$ and p-STAT3 in the DLD1-5FU-C10 cells using immunocytochemistry (Fig. 6). The immunocytochemistry results showed that p-STAT3 signaling was increased in the chemoresistant human CRC cells. 


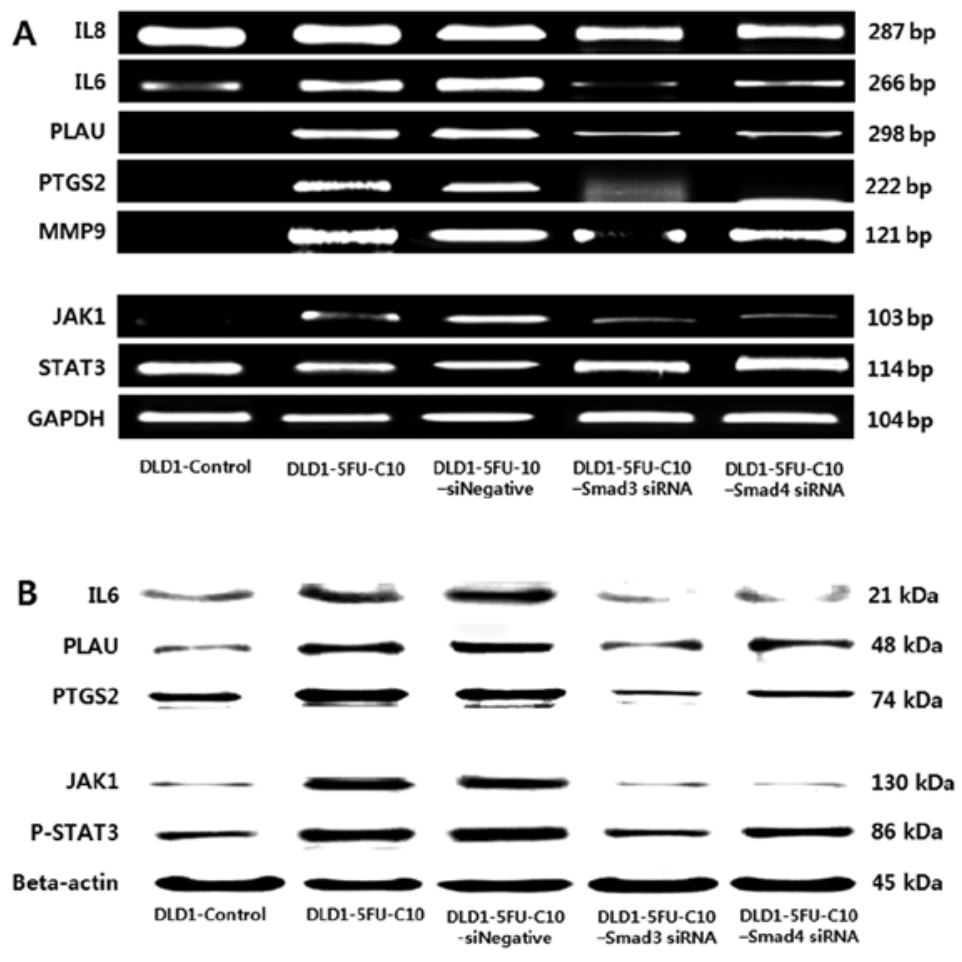

Figure 5. Effect of Smad3/4 on 5 TGF $\beta$ downstream cytokines and the JAK/STAT pathway in low drug sensitivity human colorectal cancer (CRC) cells. Smad3/4 affected 5 TGF $\beta$ downstream cytokines and the JAK/STAT pathway in 5 groups of DLD-1 CRC cells (DLD-1 control, DLD1-5FU-C10, DLD1-5FUC10-Negative siRNA, DLD1-5FU-C10-Smad3 siRNA, DLD1-5FU-C10-Smad4 siRNA). (A) Reverse transcription-polymerase chain reaction (RT-PCR) of the expression of 5 TGF $\beta$ downstream cytokines (IL8, IL6, PLAU, PTGS2 and MMP-9) and the JAK1/STAT3 pathway (JAK1 and STAT3) in the 5 groups of DLD1 CRC cells. (B) Immunoblotting analysis of the expression of 3 TGF $\beta$ downstream cytokines (IL6, PLAU and PTGS2) and the JAK1/STAT3 pathway in the 5 groups of DLD1 CRC cells. The same lysates were also used to evaluate the expression of $\beta$-actin as a loading control. IL, interleukin; PLAU, plasminogen activator; PTGS2, prostaglandin endoperoxide synthase 2; MMP-9, matrix metalloproteinase-9.
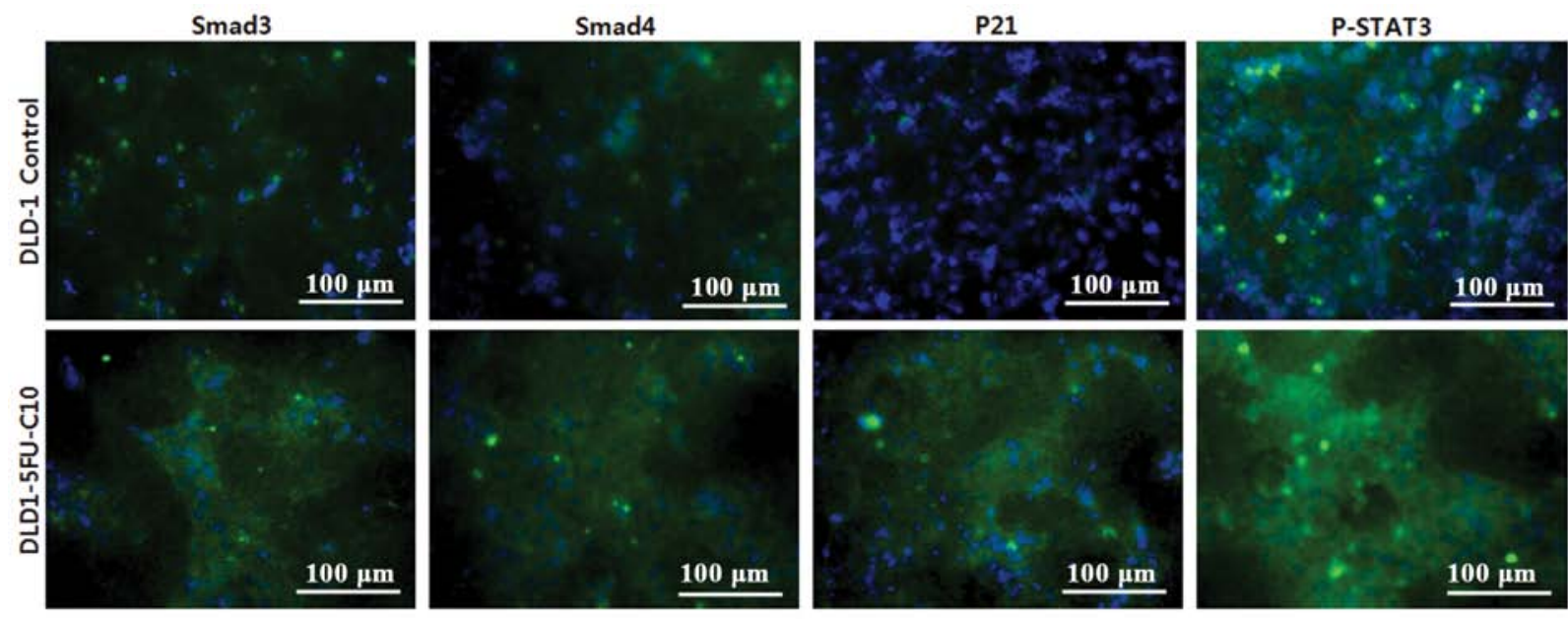

Figure 6. Expression of p-STAT3 and its related proteins (Smad3/4 and p21) in low drug sensitivity colorectal cancer (CRC) cells by immunocytochemistry. Immunocytochemical analysis of Smad3, Smad4, p21 and p-STAT3 (green) was performed in CRC cells (DLD-1 and DLD1-5FU-C10). DAPI staining is shown in blue to indicate nuclei. Magnification, $\mathrm{x} 100$. Scale bars, $100 \mu \mathrm{m}$.

\section{Discussion}

Smads are a class of proteins that function as intracellular signaling effectors for TGF $\beta$ signaling (18). Smad2 and Smad3 are activated by activin and TGF $\beta$ receptors, whereas Smad4 is activated by cytokine receptors similar to the JAK/STAT signal transduction pathway (18). In addition, a study by Dai et al showed that Smad3/4 interact with p21 and are associated with the poor prognosis of breast cancer patients $(15,25,26)$. However, the effect of Smad3/4 on drug sensitivity in CRC has not yet been established. In the present study, we investigated the role of Smad3/4 in the drug sensitivity of CRC cells with TGF $\beta$-mediated resistance to 5 -FU.

TGF $\beta$ is known to be a regulating factor in many types of cancers. Following ligand-binding, the TGF $\beta$ receptor is activated and phosphorylates 2 cognate Smads, Smad2 and 
Smad3, which then bind to Smad4. The resulting complex then translocates to the nucleus and regulates the expression of many genes by binding to their promoters (23). Previous studies have raised various questions regarding when the TGF $\beta$ signaling pathway switches from tumor suppression to tumor propagation (27).

Here, we found that the chemoresistant CRC cell line DLD1-5FU-C10 was resistant to growth inhibition. In particular, we found that high $\mathrm{Smad} 3 / 4$ expression was required for cell proliferation and migration in the TGF $\beta$-mediated chemoresistant CRC cells (DLD1-5FU-C10). In agreement with these results, Smad3/4 knockdown using siRNA significantly decreased tumor propagation and migration in the anticancer drug environment (Figs. 3 and 4). Collectively, these findings support the notion of a chemotherapy-resistant pathway to Smad3/4 in CRC, in accordance with the results of a previous study on breast cancer (15). The breast cancer study reported that Smad3/4 pro-migratory functions are mediated by $\mathrm{p} 21$ and that 5 major cytokines (IL6, IL8, PLAU, MMP-9 and PTGS2) were induced (15). In the present study, we showed that in the DLD1-5FU-C10 cells the protein levels of 3 cytokines (IL6, PLAU and PTGS2) were increased by Smad3/4 and p21 (Fig. 5A).

Studies have shown that IL6, secreted by lamina propria $\mathrm{T}$ cells and macrophages, activated the JAK/STAT pathway and promoted proliferation of tumor cells in a murine CRC model $(28,29)$. In addition, a study by Lee et al showed that p-STAT3 signaling decreased anticancer drug sensitivity in human glioma cells (30). Our results showed that Smad3/4 turns on the JAK1/STAT3 pathway via IL6, since IL6 expression is controlled by Smad3/4 (Fig. 5B). Moreover, both cytoplasmic Smad3/4 and p21 were consequently increased in p-STAT3 signaling (Fig. 6). Therefore, Smad3/4 have anti-apoptotic effects in the anticancer drug environment, which modulate the gene transcription of several TGF $\beta$ downstream cytokine genes (IL6, PLAU and PTGS2). In particular, high expression of PLAU, which is important for invasive growth, contributes to distinct aspects of cellular transformation.

However, we do not know exactly how PLAU and PTGS2 function in conferring resistance to chemotherapy in CRC. It is important to investigate whether Smad3/4-induced PLAU or PTGS2 is correlated with any other pathways. Moreover, our experimental results was limited to only one CRC cell line and anticancer drug, and our resistant clone was made drug-resistant by TGF $\beta$. Our results suggest that Smad3/4 act as regulators of chemoresistance in TGF $\beta$ mediated chemoresistant CRC cells and the association between Smad3/4 and p21 is related to the JAK1/STAT3 pathway.

To summarize, we described the role of Smad3/4 in chemoresistant CRC cells via p21. We showed that Smad3/4 interact with p21 and regulate p-STAT3 signaling by IL6. In addition, we identified Smad3/4 as a key factor in the JAK1/STAT3 pathway in chemoresistant CRC progression in an anticancer environment. Finally, these results highlight an important role for Smad3/4 signaling in anticancer drug sensitivity in CRC.

\section{Acknowledgements}

This study was supported by a grant (no. 02-2013-012) from the SNUBH Research Fund. The authors thank J. Patrick Barron,
Professor Emeritus, Tokyo Medical University and Adjunct Professor, Seoul National University Bundang Hospital for his editing of this manuscript.

\section{References}

1. Hanahan D and Weinberg RA: Hallmarks of cancer: the next generation. Cell 144: 646-674, 2011.

2. Siegel R, Naishadham D and Jemal A: Cancer statistics, 2013. CA Cancer J Clin 63: 11-30, 2013.

3. Wels J, Kaplan RN, Rafii S and Lyden D: Migratory neighbors and distant invaders: tumor-associated niche cells. Genes Dev 22: 559-574, 2008.

4. Derynck R and Feng XH: TGF-beta receptor signaling. Biochim Biophys Acta 1333: F105-F150, 1997.

5. Massague J: TGF-beta signal transduction. Annu Rev Biochem 67: 753-791, 1998.

6. Massague $\mathrm{J}$ : The transforming growth factor-beta family. Annu Rev Cell Biol 6: 597-641, 1990.

7. Roberts AB, Anzano MA, Lamb LC, Smith JM and Sporn MB: New class of transforming growth factors potentiated by epidermal growth factor: isolation from non-neoplastic tissues. Proc Natl Acad Sci USA 78: 5339-5343, 1981

8. Sinha S, Nevett C, Shuttleworth CA and Kielty CM: Cellular and extracellular biology of the latent transforming growth factor-beta binding proteins. Matrix Biol 17: 529-545, 1998.

9. Chen CR, Kang Y and Massague J: Defective repression of c-myc in breast cancer cells: A loss at the core of the transforming growth factor beta growth arrest program. Proc Natl Acad Sci USA 98: 992-999, 2001.

10. Derynck R, AkhurstRJ and Balmain A: TGF-beta signaling in tumor suppression and cancer progression. Nat Genet 29: 117-129, 2001.

11. Akhurst RJ and Derynck R: TGF-beta signaling in cancer - a double-edged sword. Trends Cell Biol 11: S44-S51, 2001.

12. Wakefield LM and Roberts AB: TGF-beta signaling: positive and negative effects on tumorigenesis. Curr Opin Genet Dev 12: 22-29, 2002.

13. Tang B, Vu M, Booker T, et al: TGF-beta switches from tumor suppressor to prometastatic factor in a model of breast cancer progression. J Clin Invest 112: 1116-1124, 2003.

14. Gong J, Ammanamanchi S, Ko TC and Brattain MG: Transforming growth factor beta 1 increases the stability of p21/WAF1/CIP1 protein and inhibits CDK2 kinase activity in human colon carcinoma FET cells. Cancer Res 63: 3340-3346, 2003.

15. Dai M, Al-Odaini AA, Arakelian A, Rabbani SA, Ali S and Lebrun JJ: A novel function for p21Cip1 and acetyltransferase $\mathrm{p} / \mathrm{CAF}$ as critical transcriptional regulators of TGFbeta-mediated breast cancer cell migration and invasion. Breast Cancer Res 14: R127, 2012.

16. Tsushima H, Kawata S, Tamura $\mathrm{S}$, et al: High levels of transforming growth factor beta 1 in patients with colorectal cancer: association with disease progression. Gastroenterology 110: 375-382, 1996.

17. Sim SH, Kang MH, Kim YJ, et al: $\mathrm{P} 21$ and CD166 as predictive markers of poor response and outcome after fluorouracil-based chemoradiotherapy for the patients with rectal cancer. BMC Cancer 14: 241, 2014.

18. Derynck R, Zhang Y and Feng XH: Smads: transcriptional activators of TGF-beta responses. Cell 95: 737-740, 1998

19. Miyazono K, ten Dijke P and Heldin CH: TGF-beta signaling by Smad proteins. Adv Immunol 75: 115-157, 2000.

20. Montgomery E, Goggins M, Zhou S, et al: Nuclear localization of Dpc4 (Madh4, Smad4) in colorectal carcinomas and relation to mismatch repair/transforming growth factor-beta receptor defects. Am J Pathol 158: 537-542, 2001.

21. Takenoshita S, Tani M, Mogi A, et al: Mutation analysis of the Smad2 gene in human colon cancers using genomic DNA and intron primers. Carcinogenesis 19: 803-807, 1998.

22. Xu J and Attisano L: Mutations in the tumor suppressors Smad2 and Smad4 inactivate transforming growth factor beta signaling by targeting Smads to the ubiquitin-proteasome pathway. Proc Natl Acad Sci USA 97: 4820-4825, 2000.

23. Massague J, SeoaneJ and Wotton D: Smad transcription factors. Genes Dev 19: 2783-2810, 2005. 
24. UlloaL, DoodyJ and Massague J: Inhibition of transforming growth factor-beta/SMAD signalling by the interferon-gamma/STAT pathway. Nature 397: 710-713, 1999.

25. Xia W, Chen JS, Zhou X, et al: Phosphorylation/cytoplasmic localization of p21Cip1/WAF1 is associated with HER2/neu overexpression and provides a novel combination predictor for poor prognosis in breast cancer patients. Clin Cancer Res 10: 3815-3824, 2004.

26. Lee $\mathrm{S}$ and Helfman DM: Cytoplasmic p21Cip1 is involved in Ras-induced inhibition of the ROCK/LIMK/ cofilin pathway. J Biol Chem 279: 1885-1891, 2004.

27. Lampropoulos P, Zizi-Sermpetzoglou A, Rizos S, Kostakis A, Nikiteas $\mathrm{N}$ and Papavassiliou AG: TGF-beta signalling in colon carcinogenesis. Cancer Lett 314: 1-7, 2012.
28. Becker C, Fantini MC, Schramm C, et al: TGF-beta suppresses tumor progression in colon cancer by inhibition of IL-6 trans-signaling. Immunity 21: 491-501, 2004.

29. Zhong Z, Wen Z and Darnell JE Jr: Stat3: a STAT family member activated by tyrosine phosphorylation in response to epidermal growth factor and interleukin-6. Science 264: 95-98, 1994.

30. Lee ES, Ko KK, Joe YA, Kang SG and Hong YK: Inhibition of STAT3 reverses drug resistance acquired in temozolomide-resistant human glioma cells. Oncol Lett 2: 115-121, 2011. 